\title{
CULTURA DE INOVAÇÃO NA EDUCAÇÃO A DISTÂNCIA E EM REDE
}

\author{
Paulo Maria DIAS ${ }^{i}$
}

\begin{abstract}
RESUMO
No presente texto desenvolvemos uma abordagem de natureza teórica e reflexiva sobre a educação a distância e em rede na sociedade digital contemporânea, tendo como referência inicial o processo de acolhimento enquanto condição para o desenvolvimento da colaboração na aprendizagem, a qual consideramos constituir a expressão central na construção do pensamento para a promoção da cultura de inovação na pedagogia e para a educação a distância e em rede, em particular, na formação ao longo da vida. O nosso objeto é promover a sustentabilidade do pensamento educacional para as cenarizações do futuro. A educação em rede constitui o foco de análise que procuramos desenvolver não só a partir das dimensões do acolhimento, mas também da partilha, da colaboração e da aprendizagem emocional e em rede, nomeadamente para a valorização da cultura de inovação nas comunidades e contextos de aprendizagem na sociedade digital.
\end{abstract}

PALAVRAS-CHAVE: Cultura de inovação, Acolhimento, Educação em rede, Partilha, Colaboração.

\section{CULTURE OF INNOVATION IN DISTANCE AND NETWORK EDUCATION}

\begin{abstract}
In this paper we develop a theoretical and reflective approach to distance and networked education in contemporary digital society, having as an initial reference the welcoming process as a condition for the development of collaboration in learning, which we consider to be the central expression to the construction of thinking to promote a culture of innovation to the pedagogy of distance and online education, in particular, in lifelong training. Our object is to promote the sustainability of educational thinking in the scenarios of the future. Networked education is the focus of analysis that we tried to develop not only from the dimensions of welcoming, but also from sharing, collaboration and emotional and networked learning, namely for valuing the culture of innovation in communities and learning contexts in the digital society.
\end{abstract}

KEYWORDS: Culture of innovation, Welcoming, Networked education, Sharing, Collaboration.

\footnotetext{
${ }^{\text {i }}$ Doutorado em Educação pela Universidade do Minho. Professor catedrático e ex-reitor da Universidade Aberta - Portugal. E-mail: paulodias@uab.pt. 


\section{CULTURA DE INNOVACIÓN EN EDUCACIÓN A DISTANCIA Y EN RED}

\section{RESUMEN}

En este texto desarrollamos una aproximación teórica y reflexiva a la educación a distancia y en red en la sociedad digital contemporánea, con la referencia inicial al proceso de acogida como condición para el desarrollo de la colaboración en el aprendizaje, que consideramos la expresión central en construcción del pensamento para la promoción de una cultura de innovación en pedagogía y para la educación a distancia y en red, en particular, en la formación permanente. Nuestro objetivo es promover la sostenibilidad del pensamiento educativo para los escenarios del futuro. La educación en red es el foco de análisis que buscamos desarrollar no solo desde las dimensiones de acogida, sino también desde el compartir, la colaboración y el aprendizaje emocional y en red, es decir, para valorar la cultura de la innovación en las comunidades y contextos de aprendizaje en la sociedad digital.

PALABRAS CLAVES: Cultura de innovación, Recepción, Educación en red, Compartir, Colaboración.

\section{INTRODUÇÃO}

Aprender em rede constitui uma expressão assumida pela comunidade da educação para descrever um modelo de ação realizado de modo não presencial. Nem sempre da melhor forma, pois encontramos uma variedade de sentidos que só se justificam pela vontade de estabelecer a inovação com foco na tecnologia, como suporte para enriquecer os ambientes de aprendizagem, em particular, na mediação dos processos da interação de comunicação.

Utilizar as tecnologias de informação para valorizar ou enriquecer os processos de aprendizagem tem uma ténue ligação com a aprendizagem em rede, pois são procedimentos centrados no espaço da sala de aula. As tecnologias são, na sua essência, instrumentos para a mediação da comunicação educativa. E o passo seguinte, que teremos de assumir, será construído na mediação pedagógica. Esta tem como objetivo construir a emergência do conhecimento e do saber fazer no coletivo enquanto princípio de coerência da comunidade, como referem Osberg \& Biesta (2020). A mediação pedagógica tem a sua sustentabilidade nos contextos de aprendizagem e na sua adequação às necessidades individuais como experiência conversacional e colaborativa do conhecimento em rede.

Por outro lado, a interação tecnológica, seja nas formas simples da comunicação direta interpares ou nas mais avançadas com o suporte da inteligência artificial, (esta última com o recurso a sistemas virtuais como os ambientes de imersão, laboratórios virtuais e sistemas de acompanhamento), tem por base a tecnologia como apoio. Repito a palavra: "apoio"; pois é 
disso mesmo que se trata na complexidade do diálogo que suporta as novas dimensões de aprendizagem em rede.

A aprendizagem tem uma forte dimensão emocional que encontra referências na literatura sobre o tema em diferentes autores, entre os quais destacamos Wenger (1988) e Damásio (2017). O primeiro fala-nos das comunidades de prática como modelo de desenvolvimento organizacional, as quais, para além da dimensão de pertença ao grupo constituem um processo de interiorização das representações sociais e cognitivas das práticas da comunidade; o segundo explicita que o desenvolvimento da complexidade é a base para a construção da consciência e da representação dos estados internos dos organismos vivos e da sua interação com o mundo.

Trazemos estas referências por duas ordens de razões: a primeira, porque se prende com o modelo de formação da comunidade; a segunda, porque afirma a "consciência" como um estado de representação que é essencial na construção da identidade da comunidade.

Em reflexões anteriores sobre esta temática (Dias, 2013) considerámos a colaboração como elemento base para a pertença à comunidade. Mas entendemos hoje que, para além desta, a comunidade tem um primeiro processo de formação que consiste no acolhimento. Na natureza deste momento estamos perante um quase-encontro que se afirma na partilha, por outras palavras, na participação na comunidade de prática ainda como recém-chegado. $\mathrm{O}$ acolhimento sempre foi um tema de reflexão no pensamento social. Acolher é a primeira forma de construir a aproximação para fazer-parte-da-comunidade. Nas palavras de Derrida (2003), as quais retomamos, a "hospitalidade" enquadra a dimensão do acolhimento. Mas a "hospitalidade" descreve o acolhimento do estrangeiro, daquele que vem. A questão central é que a participação digital não constitui necessariamente a expressão do acolhimento. Deste modo, a sociedade digital não é ainda uma comunidade, mas a expressão da complexidade dos mais variados grupos de interesses e pensamento. E, nessas condições, a sociedade digital tende a ser profundamente não inclusiva pois, nas suas práticas, replica, mesmo sob formas aparentemente inovadoras, os domínios da representação da geografia cultural que tardamos em ultrapassar.

Neste sentido, o acolher no digital não é ainda uma expressão da inclusão, mas sim uma agregação a qual não tem ainda o sentido ético para a construção da colaboração em rede, tópico 
que agora retomamos do pensamento de Palloff \& Pratt (2005) para o desenvolvimento da "comunidade de colaboração".

Deste modo, entendemos que a sociedade digital contemporânea representa ainda uma expressão mimética da cultura e expressão do território e da soberania sustentada no poder da força sobre o outro, pois tem como base não um acolhimento, mas a relação com o outro como adversário, num exercício de recusa e auto-imunologia, como refere Byung-Chul Han (2019).

Por esta razão, as propostas de inovação em educação apresentam, com recorrência, uma curva de insucesso após a sua aplicação, apesar de a inovação na educação constituir o grande motor para o desenvolvimento do conhecimento e o bem-estar social e físico da comunidade aprendente na sociedade digital.

Deste modo, o digital desenvolve-se ainda no dilema entre a narrativa do território e a utopia do hipertexto, termo cunhado por Ted Nelson (1981), na hipercultura da globalização ainda em construção, em grande parte pelas reservas ao modelo de sociedade aberta e em rede.

Temos de interiorizar que a narrativa da aprendizagem e conhecimento em rede é absolutamente inovadora, em particular, no domínio da educação inclusiva. Não basta produzirmos conteúdos digitais mantendo a matriz discursiva do presencial na hiperculturalidade, mas é necessário procurar e entender como devemos construir as narrativas na sociedade de educação aberta para o acolhimento e a aprendizagem, sendo estes os princípios da mediação pedagógica orientada para a emergência do conhecimento na comunidade em rede.

A mediação pedagógica constitui assim o modelo para a construção do diálogo na aprendizagem e criação do conhecimento orientado para a valorização dos processos colaborativos na educação aberta e em rede.

\section{A EXPRESSÃO DA INCLUSÃO}

Permitimo-nos um novo olhar sobre o acolhimento na comunidade em rede: a expressão da inclusão. Deste modo, podemos dizer que o acolhimento é a forma de construir a educação aberta e em rede, pois é o momento de partilha para a sustentabilidade do pensamento construído em colaboração. 
Mas o que é necessário referir é que acolher constitui o sentido para receber o que é diferente, porque vem de um outro lugar, e, deste modo, enriquece o património social e cultural da comunidade que o acolhe. Aquele que acolhe afirma a maior liberdade para si mesmo e para o outro, o acolhido, pois recebe-o em si e na sua comunidade. Ao acolher, está a conduzi-lo para uma nova comunidade, a da sua pertença, que assim será também a do acolhido.

Como poderemos transformar a sociedade digital numa sociedade de acolhimento? Esta é a pergunta que devemos fazer. Entendemos que a sociedade digital é onde acontece a presença virtual e não ainda o acolhimento, tal qual se apresenta nas atuais formas de participação e nas práticas de utilização das redes sociais. Assim o digital é um espaço no qual todos podemos participar desde que tenhamos uma ligação à rede - condição básica para a participação no conhecimento emergente na sociedade digital. Mas isto não evita a exclusão para quem não tiver literacia digital, social ou cultural, sendo que a maior expressão da exclusão se manifesta na arrogância da soberania sustentada na representação da geografia cultural.

Assim a condição de não acesso, seja pela literacia ou pela tecnologia, transforma-a numa sociedade da exclusão e, deste modo, numa sociedade de rejeição da inovação no pensamento social. Sobre este tema Ventiglimia \& Pullman (2016) referem, em particular, que a literacia digital permite novas formas de pensamento as quais não são concretizadas através da expressão na literacia tradicional. Acrescentamos que a literacia digital está ainda em fase de desenvolvimento, sendo, de um modo geral, construída com base na literacia tradicional baseada na transferência do modelo presencial para a esfera do virtual.

Nesta perspetiva, o digital está ainda longe de afirmar a expressão do acolhimento, pois segue o padrão do presencial. O sentido do desenvolvimento do conhecimento em rede está muito para além da participação que se afirma ainda como uma presença, para desenvolver um outro modo de interagir na colaboração no seio da comunidade.

Participar constitui, de um modo geral, o estar em rede e afirmar uma presença, mas sem apresentar necessariamente um contributo para a criação de conhecimento e valor para a comunidade. Em princípio, participar é condição de desenvolvimento para a criação de informação mas, como sabemos, esta informação representa dados e não a expressão do conhecimento. A informação só se transforma em conhecimento quando a trabalhamos até produzir um sentido, como uma representação cognitiva, um mapa mental ou de ação. O que 
sabemos dos estudos conduzidos nas neurociências (Damásio: 2017) é que o mapeamento do organismo constitui o processo de recolha da informação, mas esta só é válida para a tomada de decisão quando organizada num programa de ação do organismo.

O mapa de ação é uma expressão na construção-criação da narrativa que traz a razão para si mesmo, para a interioridade da sua expressão. Na construção deste discurso a palavra desenha o mapeamento das expressões para a criação do universo da representação da alteridade. Esta é a expressão da cosmologia das vozes dos mundos vividos e construídos em palavras e sons. Pois a narrativa constitui, em primeiro lugar, a expressão da representação para fazer-a-comunidade de partilha do pensamento e dos contextos, os quais Figueiredo (2016) apresenta como os momentos/espaços de construção da experiência do conhecimento.

Por outras palavras, a narrativa é a expressão da voz social e cultural, da comunidade e do seu pensamento, na medida em que procura, através da representação, construir o social na expressão dos mundos de experiência, como Étienne Wenger (1998) elaborou no seu pensamento crítico sobre as comunidades de prática.

Neste sentido, as comunidades de prática constituem ainda a expressão da participação, e o percurso a desenvolver na educação a distância e aprendizagem em rede, será transformálas em comunidades de acolhimento.

Entendemos que o acolhimento encontra na narrativa, em particular, nas dimensões cultural e social, a expressão mais intensa da voz-em-construção, a voz da comunidade que se expande do local para o global, do interior para o exterior, do privado para o partilhado e em colaboração no espaço público, o espaço da comunidade.

A narrativa da educação aberta encontra na voz social o sentido para transformar o local numa expressão para a construção do pensamento global. E é este o sentido que devemos perseguir para além da presença no processo de ação dinâmico de construção da teia da partilha, enquanto procedimento para a expressão da voz como ação para a construção social da comunidade inclusiva, aberta e em rede. 


\section{O DIGITAL HOJE}

A sociedade digital contemporânea apresenta uma expressão mimética na cultura e na representação do território e da soberania sustentada no poder da força sobre o outro, pois tem como base não um acolhimento, mas a relação com o outro, o que vem-de-um-outro-lugar, num exercício de confronto. Esta é a expressão da negatividade que recusa a liberdade na criação do conhecimento como expressão de soberania na sociedade em rede.

A negatividade constitui a recusa de abertura à mudança e, quando expressa na rede, transforma-a num espaço fechado em si mesmo: para a inovação, a mudança e o acolhimento. No limite, a rede representa um número de participantes, mas não a forma da sua intervenção, pois participar é ainda ser um ator. O mais importante é transformar este ator num autor, enquanto condição necessária para o desenvolvimento dos contextos de aprendizagem e conhecimento em rede.

Ser autor constitui o sentido maior para a construção de uma rede de conhecimento aberta a qual tem como fundamento a colaboração, como primeira forma para o desenvolvimento da experiência do conhecimento.

A negatividade é a expressão de uma sociedade construída na normatividade, como refere Byung-Chulk Han (2019) e, em consequência, no desenvolvimento da educação baseada essencialmente nos modelos e processos de reprodução social e cultural.

Mas, se é importante preservar os cânones enquanto corpo de referências, mais importante ainda é promover o pensamento para a descoberta, na positividade, pois os objetivos da sociedade de hoje, nos domínios culturais, sociais, económicos e tecnológicos serão absolutamente diferentes amanhã. O padrão de desempenho de hoje estará totalmente desajustado à realidade do futuro se pensarmos que as profissões de amanhã serão, em grande parte, desconhecidas e, assim, devemos escolarizar os nossos estudantes, desde a educação préescolar à universidade, para viverem nesse futuro e num modelo de formação e educação ao longo da vida.

A sociedade líquida constitui, no pensamento de Bauman (2005) e na leitura crítica de Byung-Chul Han (2019), a representação da positividade extrema. Na voz destes autores a positividade representa uma certa forma de desregulação, através da passagem abrupta da 
normatividade para um espaço aberto e, deste modo, líquido. A positividade extrema está ainda baseada no desempenho como ator num percurso sem limites, na sociedade em rede, que conduz à exaustão individual e social porque se esgota na performatividade.

Contudo, entendemos que a sociedade líquida faz parte do percurso que estamos a desenvolver para o desenvolvimento da hipercultura digital e, deste modo, constitui o modelo de transição entre a negatividade e a positividade produtiva do pensamento social, cultural e tecnológico na globalização da educação. Por outras palavras, este é o meio para a construção da sociedade do acolhimento e conhecimento em rede.

A sociedade líquida esbateu, aparentemente, as fronteiras na rede digital, mas não o espelhou nas comunidades físicas, pois estas têm como fundamento a pertença que sobrevive ainda na expressão do território.

Como melhor exemplo sobre esta matéria referimos que todos participamos na sociedade em rede sem nada acrescentar, de forma significativa, para além da presença virtual que caracteriza o modelo líquido. E a representação digital do território continua ainda a ser uma participação sem a dimensão do acolhimento.

Participar é ainda uma herança da presença que tem origem no pensamento da sociedade industrial, através do desempenho nos papéis atribuídos a cada um, enquanto atores, na educação orientada pelo pensamento da negatividade e no princípio da reprodução (Robinson: 2009).

\section{EXPRESSÃO DA PARTILHA NA EDUCAÇÃO DIGITAL}

O conhecimento em rede terá de se afirmar na sociedade do acolhimento como expressão da partilha na educação digital, a qual trazemos para esta discussão. O acolhimento é a primeira forma de abertura da educação para a inovação. A educação baseada no acolhimento não pode ser desenvolvida num modelo de reprodução, pois integra na sua essência o saber receber sem limites de natureza cultural ou social. Receber significa abrir o nosso espaço para aquele-que-vem. A comunidade de prática apresentada por Wenger (1998) é uma forma inicial de participação cujo desenvolvimento está orientado para o processo de acolhimento e integração na cultura do grupo. A comunidade de prática constitui, deste modo, 
a expressão maior do acolhimento nas práticas da educação digital, pois tem como base não só o acesso, através da participação, mas, de um modo maior e mais explícito, o pensamento do acolhimento na configuração dos processos de colaboração para a formação da identidade do grupo.

Este é o grande dilema para o pensamento curricular a ser desenvolvido num modelo normativo ou de liberdade, de reprodução ou de inovação.

Que conhecimento queremos construir na sociedade em rede? Normativo ou de inovação?

Ao optarmos pelo modelo normativo, só precisamos de aplicar as tecnologias para a promoção dos processos de aprendizagem, sem preocupações maiores com a pedagogia. $\mathrm{Na}$ verdade, esta tem vindo a ser a linha de desenvolvimento mais seguida, nomeadamente, através da incessante procura de soluções de majoração dos cenários e ambientes de ensino, como os desenvolvidos pelos ambientes abertos e massivos, os quais deverão ser considerados principalmente como recursos abertos. A educação é um processo social e cognitivo baseado no modelo conversacional, na participação, partilha e colaboração entre pares. Tudo o mais constitui o conjunto de recursos educativos que podem e devem ser utilizados nestes cenários e contextos.

No processo da cultura de inovação na educação a pedagogia é central para a criação do diálogo que conduz à aprendizagem como forma de interiorização das representações, não como um simples processo de transmissão, mas como um fazer-em-conjunto para a criação do saber em comunidade e em rede.

A mudança pedagógica tem como objetivo a renovação continuada das formas do diálogo na aprendizagem e conhecimento nos cenários e contextos de antecipação; diálogo este que constitui o primeiro passo para a partilha e a construção colaborativa da experiência do conhecimento na inovação pedagógica. 


\section{EDUCAÇÃO SOCIOEMOCIONAL NA SOCIEDADE EM REDE}

Estar em comunidade é o princípio para saber-fazer o relacionamento entre pares, seja numa perspetiva de integração social ou, de outro modo, para desenvolver as interações de natureza organizacional e cultural de forma a promover o sentido de pertença ao grupo.

Este é o princípio orientador da educação socioemocional para o desenvolvimento da comunidade inclusiva na sociedade digital e em rede. Um princípio orientado para promover o aprender a fazer-em-conjunto no acolhimento e na andaimagem social e cognitiva através dos processos colaborativos. Por esta razão a emoção é decisiva para a construção do modelo de ação colaborativo e, deste modo, constitui uma perspetiva necessariamente central no desenvolvimento da educação aberta e em rede.

A educação aberta tem como princípio um currículo inclusivo que ganha a sua maior expressão nas práticas da comunidade de aprendizagem em rede. Não é um currículo orientado pelos modelos da política económica, nomeadamente na reprodução da força de trabalho, mas sim na liberdade para a criação de valor na mudança, criatividade e inovação. Deste modo, falamos de um currículo construído nos contextos sociais e cognitivos da experiência do conhecimento. Mas, sobretudo, da construção do currículo como expressão para a liberdade da aprendizagem e do conhecimento como condições para a valorização da inovação.

A liberdade pode ser uma simples abertura do espaço de ação, mas é, sem dúvida, um processo que se afirma no momento da decisão. No enquadramento da educação em rede, a liberdade é a expressão da emoção para a tomada de decisão. E a aprendizagem socioemocional tem um papel fundamental para sabermos estar na rede-do-acolhimento e da colaboração.

A educação emocional tem como objetivo promover a valorização dos processos de decisão nos momentos de interação social, os quais constituem a sustentabilidade das práticas de aprendizagem em comunidade e nos espaços de experiência colaborativa do conhecimento (Botton:2019).

Construir o conhecimento em rede é o maior desafio para o pensamento da educação e, em particular, para a teoria curricular, pois este é um processo que se desenvolve em contextos de experiência sem fronteiras entre a educação formal, não formal e informal. 
Na tradição cultural do pensamento orientador do currículo, este é desenvolvido nas dimensões política e social, e, em parte, na negatividade construída no quadro normativo de regulação. $\mathrm{O}$ que desenvolvemos até ao presente foi uma educação baseada num currículo orientado para a reprodução, com algumas matrizes atenuadoras neste processo que emergiram de iniciativas inovadoras. Mas o que precisamos de promover para enfrentar os desafios do futuro é de um currículo aberto para construir a inovação na sociedade em rede.

Na pedagogia da educação em rede enfrentamos um enorme desafio que consiste em entendermos como os modelos, processos e práticas pedagógicas estão adequados ao aprendente digital. Este é o procedimento a seguir na promoção da sustentabilidade pedagógica (Dias; 2913) que procura a valorização das práticas de aprendizagem e da experiência do conhecimento nos contextos e ambientes digitais emergentes, as quais são essenciais para o pensamento no domínio da cultura da inovação pedagógica.

O pensamento corrente sobre esta matéria tende a utilizar procedimentos de transferência do presencial para o digital, contribuindo, deste modo, para o desenvolvimento de um processo de ação que promove um momento de educação que se afigura sem sustentabilidade na sociedade digital emergente. A aprendizagem no domínio do digital em tudo é diferente do presencial. A interação é mediada tecnologicamente e a construção das aprendizagens decorre de um outro procedimento que tem como base a mediação pedagógica desenvolvida na positividade, na liberdade e orientada para a cultura de inovação, a qual implica um enorme esforço individual e um profundo domínio da autorregulação dos processos sociais e cognitivos de participação, e socialização nos contextos de criação colaborativa do conhecimento, como referem Sarah \& Castells (2019:27) ao afirmarem que "a criatividade é a capacidade para criar e produzir um novo conhecimento ou significado". Esta é a educação que temos de afirmar na sociedade do conhecimento em rede.

A educação é o acolhimento-no-diálogo para a criatividade e, por esta razão, a sensação de presença social e cognitiva da comunidade é fundamental. Mas esta perceção só se concretiza com um estudante que aceite aderir a um programa de trabalho em rede. Tecer a rede digital de conhecimento é um programa colaborativo que nada tem a ver com a tradição da aula expositiva presencial, pois esta tem na sua essência programática a exposição de conteúdos, e a rede é a 
expressão da construção colaborativa da teia da experiência do conhecimento sob a forma de uma representação hipercultural.

Entendemos assim que a exposição terá de ser transformada numa voz social para valorizar o contexto de experiência do aprendente, colocando-o no centro da criação do conhecimento. A voz comum nesta matéria afirma a aprendizagem centrada no aprendente de acordo com o modelo construtivista.

Mas o mais interessante, neste ponto da nossa reflexão, é que o modelo construtivista, apesar de profundamente inovador, emergiu no pensamento da negatividade e, o que procuramos, é uma nova expressão construída na positividade, sem o excesso da autoalienação que se revela no movimento contínuo de superação individual na sociedade da positividade extrema (Byung-Chul Han: 2019).

Nesta perspetiva, podemos observar o pensamento construtivista sob uma outra dimensão na construção social da positividade. Para o fazermos, partimos da conceção de que o construtivismo constitui um alicerce sólido para a compreensão dos processos de desenvolvimento da cognição. Mas o desenvolvimento social e cognitivo apresenta uma plasticidade emocional própria que se manifesta na evolução dos sistemas de construção e elaboração do pensamento nos contextos de ação e aprendizagem.

A construção do conhecimento em rede está para além dos princípios fundadores do pensamento construtivista que conduziu ao desenvolvimento da aprendizagem centrada no aprendente. $\mathrm{Na}$ educação em rede, a aprendizagem é um processo colaborativo realizado na partilha social e cognitiva da experiência do conhecimento nos contextos de aprendizagem. Deste modo, devemos considerar a hipótese de que os processos cognitivos e sociais da aprendizagem constituem a emergência da inteligência em rede, como afirmou Siemens (2005) na teoria do conectivismo; por outras palavras, a cognição no contexto social constitui a expressão de representação da comunidade em rede. E a rede constitui a expressão da andaimagem no desenvolvimento da representação cognitiva na comunidade de acolhimento e colaboração.

Na perspetiva do construtivismo, a cognição constitui o modelo de ação individual, contudo este é um modelo que se expande para o social através da colaboração. No modelo de 
partilha social e em rede a construção do pensamento é um processo realizado colaborativamente e sob um modelo inovador: "aprender em rede".

Com esta afirmação não desvalorizamos a importância dos processos de desenvolvimento cognitivo, mas desenvolvemos o foco na análise para o que resulta do mapeamento individual e da sua integração na comunidade, enquanto expressão da partilha na criação do conhecimento em rede.

\section{CONCLUSÃO}

Uma metáfora possível para descrever o conhecimento em rede consiste no movimento dos oceanos. No mar, que entendemos como nosso, quando a onda se derrama na areia, temos sempre um mundo que está para além da geografia na proximidade física. Esta é uma das formas para descrever a proximidade virtual que estamos a construir em rede na sociedade digital.

Os oceanos são a expressão de todos os lugares e da ligação entre todas as comunidades. A onda que se estende na areia tem a liberdade de proximidade com todos os lugares os quais se transformam assim num acolhimento.

A aprendizagem é um processo que emerge do mapeamento do contexto e a partir do qual é realizada uma representação interna no organismo vivo, a qual está ligada, por definição do desenvolvimento do processo, à rede social com a qual este organismo interage.

Neste sentido, a aprendizagem não resulta de um processo de colonização, mas pelo contrário, na expressão do acolhimento e integração na comunidade.

A diferença entre um algoritmo de aprendizagem automática e um organismo vivo é que o primeiro depende da colonização de uma base de informação a partir da qual elabora um padrão de representação sob a forma de "conhecimento virtual". O segundo, constrói a representação interna e social através da interação direta com a informação nos contextos de experiência, transformando-a em conhecimento nas dimensões individual e da comunidade, como o faz o oceano.

Este é o princípio para a cultura de criatividade e inovação que se afirma na disrupção dos padrões do conhecimento estabelecido, cujo cânone do pensamento nada mais significa, para além da referência, numa sociedade em mudança. 
A mudança é o primeiro momento para o desenvolvimento da inovação. Neste sentido, a mudança social e cognitiva constitui a expressão de todos os momentos de inovação na construção das comunidades de aprendizagem e conhecimento em rede.

Como refere Pinker (2007:280) na sua reflexão sobre os processos de desenvolvimento do pensamento e da cognição, os modelos combinatórios permitem “... que um grupo finito de ideias simples dê origem a um grupo infinito de ideias complexas". Este é o princípio da complexidade que está na origem da consciência e do que entendemos ser o desempenho inteligente nos processos de abstração e criatividade para a cultura de inovação. A representação emergente do mapeamento é absolutamente inovadora para o desenvolvimento do organismo. Os dados recolhidos, através dos processos de mapeamento, são assim transformados numa representação holística para a tomada de decisão, em particular, porque o organismo afirma o procedimento de autor no processo de desenvolvimento da interação com os contextos. De igual modo a educação a distância e em rede terá de afirmar a sustentabilidade no mapeamento dos contextos formais, não formais e informais para o acolhimento e a experiência colaborativa do conhecimento na sociedade digital.

Este é o maior desafio para promovermos a cultura de inovação na educação a distância e em rede.

\section{REFERÊNCIAS}

Bauman, Zigmunt (2005). Liquid life. Cambridge: Polity Press.

Bent-Weiser, Sarah \& Castells, Manuel (2017). Economia é Cultura. In: Manuel Castells (Org.). Outra Economia é Possível. Rio de Janeiro: Zahar Editor.

Botton, Alain de (2019). The school of life: an emotional education. Penguin Books, LTD.

Byung-Chul, Ham (2019). Hiperculturalidade, cultura e globalização. Petrópolis: Editora Vozes.

Damásio, António. (2017). A Estranha Ordem das Coisas. Lisboa: Círculo de Leitores.

Derrida, Jacques (2003). Não há passo/da hospitalidade. In: Anne Dufourmantelle \& Jacques Derrida. Da hospitalidade. Coimbra: Palimage. 
Dias, Paulo (2013). Inovação pedagógica para a sustentabilidade da educação aberta e em rede. Educação, Formação \& Tecnologias, 6 (2), 4-14 (Online). Disponível em: http://eft.educom.pt.

Figueiredo, António Dias (2016). A Pedagogia dos Contextos de Aprendizagem. $e$ Curriculum, v. 14, n. 3, 2016, p. 809-836. Disponível em: https://revistas.pucsp.br/index.php/curriculum/article/view/28989.

Nelson, Ted (1981). Literary machines. Texas. USA: author.

Osberg, Deborah \& Biesta, Gerta, (2020). Beyond curriculum. Groundwork for a noninstrumental theory of education. Educational Philosophy and Theory. Disponível em: DOI101080/00131857.2020.1750362.

Pallof, Rena \& Pratt, Keith (2005). Collaborating online: Learning together in community. San Francisco, CA: Jossey-Bass.

Pinker, Steven (2007). Do que é feito o pensamento. São Paulo: Editora Schwarcz, LTDA.

Robinson, Ken (2009). The Element. USA: Penguin Books.

Siemens, George (2005) Connectivism: A learning theory for the digital age, International Journal of Instructional Technology and Distance Learning 2 (10). Disponível em: https://jotamac.typepad.com/jotamacs_weblog/files/Connectivism.pdf.

Ventimiglia, Phil \& Pullman, George (2016). From Written to Digital: the new literacy. Educause Review, March/April, pp.36-48.

Wenger, Étienne. (1998), Communities of Practice, Learning, Meaning, and Identity. Cambridge University Press: USA.

Recebido em: 21/09/2020

Aprovado em: 15/10/2020 